\title{
Long-term Monitoring of the Sierra Nevada Snowpack Using Wireless Sensor Networks
}

\author{
Ziran Zhang, Steven Glaser, Thomas Watteyne, Senior Member, IEEE, Sami Malek
}

\begin{abstract}
Historically, the study of mountain hydrology and the water cycle has been largely observational, with meteorological forcing and hydrological variables extrapolated from a few infrequent manual measurements. Recent developments in Internet of Things (IoT) technology are revolutionizing the field of mountain hydrology. Low-power wireless sensor networks can now generate denser data in real-time and for a fraction of the cost of labor-intensive manual measurement campaigns.

The American River Hydrological Observatory (ARHO) project has deployed thirteen low-power wireless IoT networks throughout the American River basin to monitor California's snowpack. The networks feature a total of 945 environmental sensors, each reporting a reading every 15 minutes. The data reported is made available to the scientific community minutes after it is generated.

This paper provides an in-depth technical description of the ARHO project. It details the requirements and different technical options, describes the technology deployed today, and discusses the challenges associated with large-scale environmental monitoring in extreme conditions.
\end{abstract}

Index Terms-Wireless Sensor Networks, real-time, IoT, environmental monitoring, hydrology, real-world solutions, largescale deployments, extreme conditions.

\section{INTRODUCTION}

Snow-melt from the Sierra Nevada mountains constitutes more than half of the fresh water demand in the state of California. Severe droughts in the past years have motivated an increased stakeholders interest in a finer monitoring of this newly sparse resource. There is a growing need for better streamflow predictions to allow for more efficient dam operations at the reservoirs level, mainly in terms of hydropower generation, flood control and drought mitigation. Different instruments have recently gained attraction from both governmental water agencies and utilities companies, and are being employed to improve snowpack monitoring. They include remote-sensing (MODIS), aerial Light Detection and Ranging (LiDAR), insitu sensors, weather forecasts and ideally their synergistic combination. Though remote-sensing typically covers wide areas, it lacks fine spatial and temporal resolution, and thus is complemented with finer resolution limited range techniques such as LiDAR and in-situ sensors. Snowdepth measurements are currently conducted manually by surveyors where snow sampling tube were used to measure snow depths. This spatially limited, infrequent and labor-intensive method also tends to underestimate the snowpack due to unrepresentative sampling at low elevations. Less than 50 surveys are conducted

Z. Zhang, S. Malek, and S. Glaser are with UC Berkeley, CA, USA. (e-mail: \{zhangziran,sami.malek,glaser\}@ berkeley.edu).

T. Watteyne is with Inria, France. (e-mail: thomas.watteyne@inria.fr).

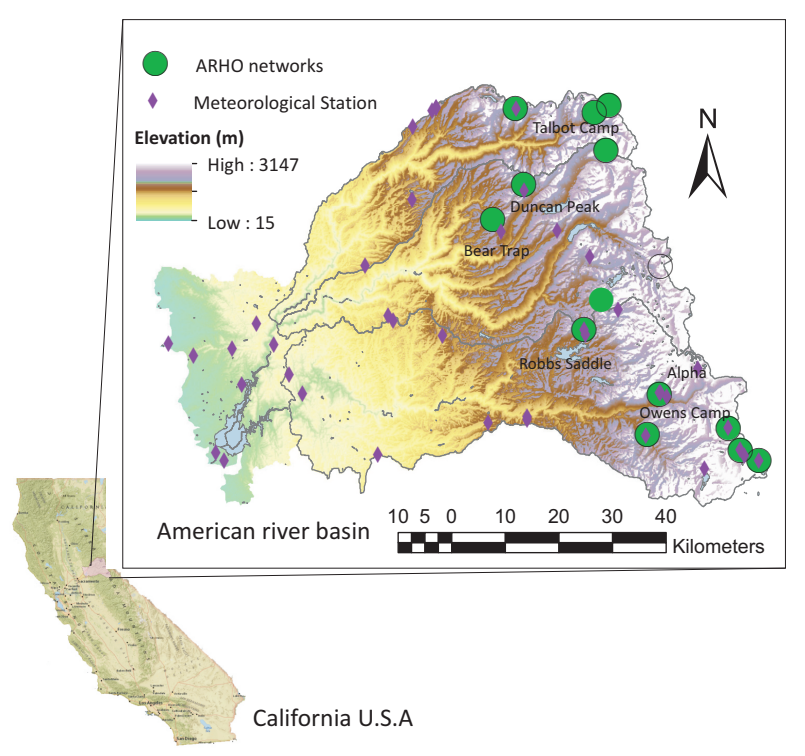

Fig. 1: 13 networks are running today in California's American River Basin. With 945 sensors, these networks offer realtime monitoring of the snow melt process of the Sierra Nevada, and is arguably the largest IoT platform deployed for environmental monitoring

for the whole $5000 \mathrm{~km}^{2}$ American River Basin, yet it has been shown that snow depth distribution is non-uniform in those areas. Finer-scale observations are therefore indispensable for a better understanding of the snowpack. A relatively small area of $1 \mathrm{~km}^{2}$ typically features diverse levels and types of vegetation, as well as intricate terrain formations that affect snow deposition and melting. Capturing this diversity requires a dense set of sensors deployed in this $1 \mathrm{~km}^{2}$ area.

The goal of the American River Hydrological Observatory (ARHO) project is to provide high spatial and temporal resolution meteorological and hydrological measurements at the American River watershed scale, and thus satisfy the high accuracy and coverage needed for optimal water resources management.

So far, we have deployed 13 low-power wireless IoT networks throughout the American River Basin that leverage the state-of-the-art, low-power wireless technologies. The oldest networks have been installed and in service since summer 2013. Each network covers approximately $1 \mathrm{~km}^{2}$ of terrain, and consists of between 30 and 50 low-power wireless "motes" 
connected to a gateway device, which in turn is connected to the Internet via a cellular or satellite modem.

Each mote is equipped with at least four environmental sensors (snow level, solar radiation, soil moisture, temperature and humidity). All sensors were calibrated at the manufacturer facilities prior to deployments. In total, we have 414 nodes deployed, hosting 945 sensors, each of which takes a reading every 15 minutes. Measurement data is pushed to the cloud in real-time, and thus is accessible to the scientific community minutes after it has been measured ${ }^{1}$. To the best of our knowdledge, the ARHO networks constitue the largest wireless sensor network deployed for environmental monitoring in the world.

This paper describes the methods and components used in such a large scale-system, as well as its reliability and scalability allowing for easy expansion from catchment (kilometer scale) to river basin (thousands of square kilometer scale) to full fledged mountain chain scales.

Across these different scales, multiple sensors must be integrated in a robust fashion in order to obtain confident spatial estimates of hydrologic phenomena. The novelty of our approach is to treat a network of sensors, deployed at the catchment and basin scales, as a single instrument, or - more precisely - an integrated basin-scale hydro-meteorological instrument cluster. The measurement strategy is to extend snowdepth measurements outwards from existing metrological sites, to capture the variability in watershed physiographic features, taking advantage of existing telemetry and measurement history. The overall strategy is to capture the critical gradients in weather, precipitation, and snow across the full elevation and latitudinal range of the American River Basin. To do this, augmentations to the meteorological and soil-moisture measurements will be needed across all of these components.

This article provides an in-depth technical description of the ARHO project. We describe how the data is collected and list the requirements for reliability, throughput, wireless connectivity and network lifetime, then describe the different options available, and finally discuss the solutions we adopted.

All presented data are taken from low-power wireless IoT networks that we have already installed and that are running today. We hope this article can be informative for both technology providers - by providing testing feedback and limitations - and environmental scientists — by describing a solution that works.

The remainder of this article is organized as follows. Section II presents the requirements associated with gathering environmental data in remote locations, and the different solutions available today. Section III describes the network in details. Section IV presents both networking and hydrological data gathered from the deployed ARHO networks. Section V discusses related work in remote environmental sensing. Section VI concludes this paper with a discussion on what works and what can be improved.

\footnotetext{
${ }^{1}$ data can be seen at http://glaser.berkeley.edu/wsn/.
}

\section{REQUIREMENTS AND OPTIONS}

Remote environmental sensing places unique requirements on the network interconnecting the sensors. Section II-A starts by listing those requirements; Section II-B then details the networking options in light of those requirements. The goal of the ARHO project is not to develop a new networking technology, but rather to "bring it all together" by integrating the appropriate off-the-shelf networking solution with environmental sensors.

\section{A. Requirements for Remote Environmental Sensing}

Where exactly to place the sensing nodes is expertly determined and depends mainly on some hydrological characteristics of the terrain. The networking technology must be able to support placement of nodes at arbitrary locations. Moreover, for the collected data to be physiographically representative, the network of sensors must be able to cover $1-2 \mathrm{~km}^{2}$, and be composed of up to 60 devices.

Sites of interest are scattered throughout the Sierra Nevada, and reaching some sites requires hours of driving through forest roads which are closed during the winter. The networks must therefore be able to run "on their own" without any mandatory user intervention. They must be able to withstand important temperature swings, self-heal in case of environmental changes, and run for several years without human intervention.

Each node must be able to report a sensor measurement every 15 minutes. Assuming a 60-node network, this translates in the network generating one packet every 15 seconds. The network must successfully and securely transport the sensors data to a gateway device [1], [2]. Moreover, a user must be offered remote monitoring capabilities, i.e. the ability to remotely assess the health of the network. Hydrologists must be able to access the data gathered by the sensors within minutes of when it was measured.

\section{B. Technological Options}

Given the requirements listed in Section II-A, this section discusses the applicability of different networking technologies.

Environmental data loggers such as the Campbell CR1000, very well know in the hydrology community, store timestamped sensor data on internal memory, which can be retrieved manually. While off-line logging does not satisfy the "real-time" requirement, it is an interesting option, at least for keeping a copy of the data locally in case of networking problems.

A first networking option is to use wired technology to interconnect the different sensors. This is the technology used by the commercial products such as Campbell. This is not an option for the ARHO project for the following reasons. First, there is significant additional cost and time associated with covering a $1.5 \mathrm{~km}^{2}$ with wires. Second, the state of California has strict rules on deploying equipment in the Sierra Nevada; interconnecting sensors using wires would be too intrusive. Finally, the weight of snow-pack, falling objects, and animal 
curiosity are likely to inflict damage to wires laying on the ground [3], [4].

Wireless appears as the technology which fits the requirement of Section II-A best. Cellular coverage on some sites is non-existent, spotty on others; equipping each sensor with a cellular modem is therefore not an option. An IEEE802.11 (low-power) WiFi network would force a star topology; given the size of the deployments, WiFi doesn't offer the range required. The range of Bluetooth and the size of piconet prevent us from using Bluetooth. Moreover, to offer a 2year battery lifetime, assuming a $17000 \mathrm{mAh}$ D-cell battery, a communicating node must draw at most $970 \mathrm{uA}$, on average.

IEEE802.15.4 [5] is a wireless technology which can satisfy the requirements. It offers a good balance between data rate (250 kbps), communication range (50-100 m outdoors) and power consumption (a radio chip consumes 5-25 mA when on, depending on the vendor). Communication protocols enable the radio to be heavily duty-cycled (enough to reach the $970 \mathrm{uA}$ on average current draw above) and form a multihop topology. The challenge is that IEEE802.15.4 is "just" the physical layer; a complete flurry of protocols, products and open-source implementations build on top of it.

Numerous IEEE802.15.4-based sensors boards exist, including the TelosB and the popular OpenMote ${ }^{2}$. These boards are "bare" and do not come pre-programmed with a proven network stack. Since the goal of the ARHO project is to use an off-the-shelve solution, such bare boards are not an option if they are not bundled with a protocol stack implementation.

ZigBee is a protocol stack which builds on top of IEEE802.15.4 radio. Although a ZigBee network is multihop and can carry the data load of our applications, it has two major shortcomings. First, routing nodes needs to leave their radio $\mathrm{ON}$ all the times, as a ZigBee network is not synchronized. This means an average current draw of 5-25mA, well above the $970 \mathrm{uA}$ limit. Second, a ZigBee network operates on a single frequency, and is therefor prone to multi-path fading and external interference. In [6] and [7], the authors discuss how this prevents ZigBee from meeting industrial requirements, and is therefore also our requirements.

Several off-the-shelf proprietary IEEE802.15.4-based products exist. One example is DigiMesh, running on the XBee platform ${ }^{3}$, but suffers from the same single-frequency limitation as ZigBee. Those products also suffers from the inability to dynamically adapt to network topology changes.

SmartMesh IP is an off-the-shelf low-power wireless networking product, developed by the Dust Networks product group at Linear Technology, which satisfies all requirements listed in Section II-A. A SmartMesh IP network is multi-hop in nature, with a single gateway device (called "manager") and up to 100 motes. The protocol stack it uses is rooted in IEEE802.15.4e Time Synchronized Channel Hopping (TSCH), a new networking technology in which nodes are tightly synchronized, and where a communication schedule orchestrates all the communication in the network [8]. This results in over $99.999 \%$ end-to-end reliability, and router nodes drawing less

\footnotetext{
2 http://www.openmote.com/

3 http://www.digi.com/technology/digimesh/
}

than $50 \mu \mathrm{A}$ on average [9]. The capacity of a network is 25 packets per second, which is well above the one packet every $15 \mathrm{~s}$ requirement of our application. Section III-B provides additional details about SmartMesh IP.

Linear Technology sells generic communication modules (a small $24 \mathrm{~mm} \times 42 \mathrm{~mm}$ board), which can be easily integrated in a complete solution. Metronome Systems ${ }^{4}$ has done this integration, and offers an ecosystem of SmartMesh IP-enabled boards, which the ARHO project is using. Section III-A details the Metronome Systems ecosystem we use.

\section{AN ARHO NETWORK}

This section describes, in detail, the hardware used in an ARHO network, the SmartMesh IP protocol stack, how sensors are integrated, how the gateway is built, how a network is deployed, and what back-end system is used.

\section{A. The Metronome Systems Ecosystem}

We use the Metronome Systems "ecosystem" of low-power wireless devices.

The Manager (Fig. 2a) is a single-board Linux computer connected to a SmartMesh IP manager module, encased in a robust and water resistance enclosure. Besides the advantage of being built to cope with the extreme Sierra Nevada weather, the Manager consumes only $50 \mathrm{~mA}$ at $5 \mathrm{~V}$ on average, much less than the raspberryPi $(700 \mathrm{~mA})$ or Arduino boards $(500 \mathrm{~mA})$. In the ARHO project, the Manager is connected to a cellular or satellite modem, as detailed in Section III-D.

The NeoMote (Fig. 2b) is the sensing heart of the ARHO network. It is composed of a SmartMesh IP module and a Cypress Programmable System on Chip (PSoC5). The SmartMesh IP module provides robust and reliable wireless networking capability, the PSoC interfacing capabilities to any sensor of actuator. The PSoC offers an array of configurable system blocks that can be dynamically added to a project for a particular application. For instance, the board can interface up to 40 analog and/or digital sensors at once, providing all analog and digital signal conditioning and excitation. The PSoC building blocks are available to a drag-and-drop interface and are reprogrammable over the radio. The NeoMote supports $3.3 \mathrm{~V}, 5 \mathrm{~V}$ and $12 \mathrm{~V}$ sensors. Interfacing with a SD-card slot provides additional storage for data and system parameters. In addition, the board is ultra-low power. The PSoC consumes $30 \mu \mathrm{A}$ at $3.3 \mathrm{~V}, 60 \mu \mathrm{A}$ when the 20-bit A/D mode is active. In the ARHO project, the NeoMote is connected to sensors and mounted on a sensor node as detailed in Section III-C.

The repeater node (Fig. 2c) consists of a SmartMesh IP mote and a carrier board with a D-cell 17 Ah battery. In the ARHO project, the repeater node is used as a relay in case the sensor nodes are spread too far apart in the deployment field, as detailed in Section III-E.

\section{B. SmartMesh IP}

A SmartMesh IP network is composed of a manager and up to 100 motes. The motes form a redundant multi-hop and self-healing wireless mesh network around the manager.

${ }^{4}$ http://metronomesystems.com/ 


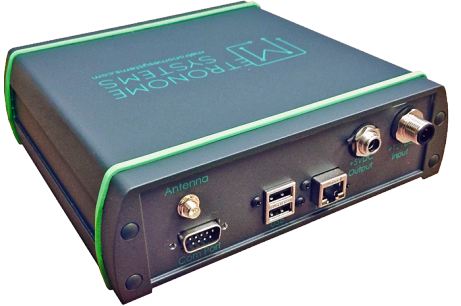

(a) Manager

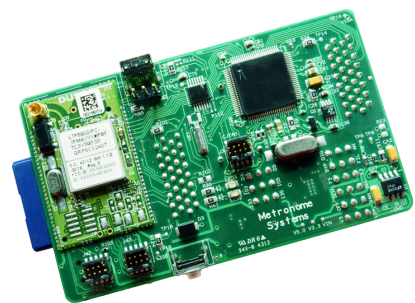

(b) NeoMote

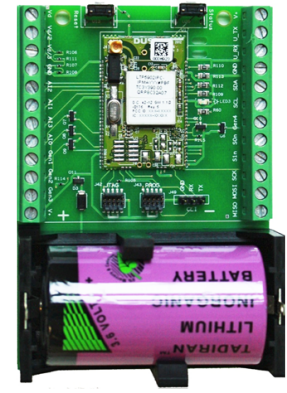

(c) repeater node

Fig. 2: The Metronome Systems hardware used in the ARHO project.

The core of a manager or mote is the LTC5800 chip, specifically designed for SmartMesh IP, and which features a ARM Cortex-M3 microntroller and an IEEE802.15.4compliant radio chip. Because it it specifically designed for SmartMesh IP, the LTC5800 features hardware acceleration for common operations, resulting in ultra low-power operation.

45,000 SmartMesh networks are deployed today, in application domains ranging from industrial process monitoring and city-wide parking solutions to building monitoring and remote sensing. SmartMesh IP provides over $99.999 \%$ endto-end reliability and less than $50 \mu \mathrm{A}$ average current draw on routing nodes, translating to over a decade of lifetime on typical batteries.

The network stack used in SmartMesh IP is rooted in the IEEE802.15.4e TSCH standards. In a TSCH network, nodes are tightly time synchronized, and time is cut into timeslots. All communication is orchestrated by a schedule which indicates to each mote what to do in each timeslot: transmit, receive or sleep. The schedule is built in such a way that nodes only switch their radio on when they actually communicate, yielding radio duty cycles well below $1 \%$. When communicating, nodes sent successive packets at different frequencies. The resulting channel hopping is known to combat external interference and multi-path fading efficiently [10][15], the two main sources of unreliability in low-power wireless networks.

The network stack used in SmartMesh IP combines the performance of TSCH (through the the IEEE802.15.4e standard) and the ease-of-use of IPv6. Each SmartMesh IP devices can be assigned a globally unique IPv6 address, significantly simplifying Internet integration. Through several sets of keying material, and the use of an AES-128 cipher, all frames exchanged in a SmartMesh IP network are secured. At any point in time, a device can be administratively removed from the network. Thanks to the underlying TSCH technology, nodes are tightly synchronized (a handful of $\mu$ s maximum de-synchronization between neighbor nodes). This means the events measured can be accurately timestamped, or that several nodes in the network can coordinate actuation.

The manager plays an important role. First, it serves as the gateway between the low-power wireless mesh and the Internet. Second, it is reponsible for building and maintaining the TSCH schedule. The manager monitors the topology of the network and the application-level communication require- ments of the nodes, and continuously adjusts the schedule accordingly. The result is that the network adapts to topological changes. Finally, the manager is responsible for coordinating the join process of new motes, which includes a security handshake, and handing out the appropriate keying material.

In a SmartMesh IP network, each node has at least 2 routing parents. In case one of the parents is switched off or removed, the second parent is used to reliably route the node's packets. To be able to exploit this path diversity, nodes need to be deployed in a sufficiently dense manner. The rule by Dust Networks is that each node should be able to "hear" at least three other nodes.

The TSCH schedule provides a clean trade-off between amount of data and power consumption. The more data a mote has to send, the more activity it will have in its communication schedule, and hence the more it consumes. Based on the communication requirements, it is possible to accurately predict the current draw of a node [9], which typically ranges between $10 \mu \mathrm{A}$ and $50 \mu \mathrm{A}$.

\section{The Sensor Node: Interfacing Sensors}

The NeoMote is the "sensing heart" of an ARHO network. Through the PSOC micro-controller, it can connect to any sensor or actuator, through digital or analog interfaces. In the ARHO project, the NeoMotes with sensors attached were enclosed in a water proof enclosure mounted on a mast. These poles, typically $5 \mathrm{~m}$ high, were secured into the ground at strategic locations throughout the deployment site.

Fig. 3 depicts such as sensor node. A waterproof box contains all the electronics, including a rechargeable battery. This box is typically mounted $2 \mathrm{~m}$ above the ground, so it can be accessed both when there is, and when there is no snow. A number of elements are mounted on a cross arm at the top of the pole. These include solar panel, as a well solar radiation, temperature, humidity and snow depth sensor. The latter is a ultrasonic range finder oriented down; it sends "chirps" and measures the time it takes for the sounds to bounce on the snow and return to its built-in microphones. Soil moisture sensors are placed at different depth in the ground to monitor the water seeping through the soil. All these elements are connected to the main electronics box through kevlar tubing, after animals, including bears, chewed and broke traditional electric wiring in early deployments. 




Fig. 3: A sensor node is composed of: (1) a NeoMote lowpower wireless mote; (2) a break out board; (3) a memory card for local data logging; (4) a rechargeable battery; (5) a snow depth sensor; (6) temperature and relative humidity sensors; (7) a pyranometer (solar radiation) sensor; (8) a solar panel; (9) an omni-directional antenna; and (10) underground volumetric soil moisture sensors.

The sensor nodes were pre-assembeld in the laboratory, and transported by truck to a deployment site. At the site, the cross arm and mast were fastened, and the pole was secuted into the ground using a U-channel. Installing a single pole takes approximately 1 hour for a team of two.

\section{The Base Station: Internet Connectivity}

The manager is attached to a sturdy 3 in diameter, $4 \mathrm{~m}$ long steel mast secured on concrete fondatation. It is very similar to sensor Node, with the different that, instead of being connected to sensors, the manager is connected to a cellular modem ${ }^{5}$ or satellite modem ${ }^{6}$. When available, we prefer cellular connectivity as it is cheaper and provides more stable services. Five sites are connected using celullar, five sites using satellite. The other three sites have their gateways yet to be installed.

The main role of this cellular/satellite connection is to upload the sensor data and network statistics onto the servers located in the back-end at UC Berkeley (see Section III-F). In addition, it allows one to log into the Linux computer of the manager remotely, to inspect the network, launch an over-theair reprogramming of the network, and update the software running on the manager.

\section{E. Deployment Strategy}

An ARHO network is composed of one manager, 10-11 sensor nodes, and a number of relay nodes in case some sensor nodes are deployed too far apart from the rest of the network to connect.

We deployment a ARHO network as follows. The locations of the sensor nodes are chosen based on their hydrological importance. In a typically deployment, the sensor nodes cover

\footnotetext{
5 Sierra Wireless RavenX

6 Inmarsat BGAN 9502
}

an area between $1 \mathrm{~km}^{2}$ and $1.5 \mathrm{~km}^{2}$. The location of the manager chosen so it has good cellular connectivity, or an open area for good satellite connectivity.

Once the sensor and gateway poles are in place, and the devices turned on, we use the monitoring tools built in the manager to verify the connectivity between the sensors. We place relay nodes in an iterative fashion, each time verifying the updated connectivity information. The rules provided by Dust Networks is for each node to have at least 3 neighbors with which it has a connection with a Received Signal Strength (RSSI) higher than $-85 \mathrm{dBm}$. We add replay nodes until that rule is satisfied. In a typical network, we add between 20 and 30 relay nodes (details in Section IV-A).

\section{F. Back-end System}

The back-end system consists of a server running at UC Berkeley, and which is responsible for receiving the data from the deployed ARHO networks, storing it in a database and presenting the data over a web interface on a first-come-firstserved basis. Each manager of an ARHO network sends the sensor data and topological information to the back-end server, using the cellular of satellite connection, periodically. Sensor data is also stored locally on each NeoMote's internal memory as backup.

\section{EXPERIMENTAL RESULTS}

This section presents the results of the ARHO project, both in terms of successfully deploying state-of-the-art low-power wireless network, and in terms of generating a large datasets useful for the hydrological community.

\section{A. Instrumenting the American River Basin}

Table I summarizes the equipment deployed to date. Thirteen networks are fully operational, some since 2013, others since 2014, and are composed of 414 low-power wireless devices, networking 945 sensors. Each day, each sensor produces 96 data records. Between August 2014 and May 2015, this resulted in 11 million sensor reading. In the same period, the ARHO networks produces $2.5 \mathrm{~GB}$ of generated data, $400 \mathrm{MB}$ of which are sensor readings, the remaining being network statistics and connectivity data. The raw data is presented in real-time through a web interface ${ }^{7}$. This raw data is turned into a weekly hydrological reports available online ${ }^{8}$.

\section{B. Lessons Learned from Deploying Networks}

The monitoring built into SmartMesh IP have proven essential to assess the health of the networks and make changes to the topology. Each node in the network publishes statistics every $5 \mathrm{~min}$, which include the number of packets it has transmitted, and the neighbors it communicates with. This information allows us to identify weak points in the topology, and add/move relay nodes accordingly, using the approach described in [16].

\footnotetext{
${ }^{7}$ http://glaser.berkeley.edu/wsn/

8 http://arho.org/
} 


\begin{tabular}{|l|c|r|r|r|}
\hline Name & Operational Since & Elevation (m) & $\begin{array}{r}\text { \# Devices } \\
\text { (Managers/NeoMotes/Relays) }\end{array}$ & \\
\hline Alpha & 2013 & 2269 & $37(1 / 10 / 27)$ & 70 \\
Bear Trap & 2013 & 1518 & $38(1 / 10 / 27)$ & 70 \\
Caples Lake & 2013 & 2437 & $37(1 / 11 / 25)$ & 79 \\
Dolly Rice & 2014 & 1980 & $11(1 / 10 / 0)$ & 70 \\
Duncan Peak & 2013 & 2907 & $42(1 / 11 / 30)$ & 78 \\
Echo Peak & 2013 & 2478 & $54(1 / 10 / 43)$ & 71 \\
Mount Lincoln & 2013 & 2477 & $36(1 / 11 / 24)$ & 78 \\
Onion Creek & 2013 & 1891 & $11(1 / 10 / 0)$ & 72 \\
Owens Camp & 2014 & 1586 & $33(1 / 11 / 12)$ & 77 \\
Robbs Saddle & 2013 & 2673 & $38(1 / 10 / 22)$ & 70 \\
Schneiders & 2013 & 1738 & $11(1 / 10 / 27)$ & 70 \\
Talbot Camp & 2014 & 2069 & $38(1 / 10 / 27)$ & 70 \\
Van Vleck & 2013 & & $414(11 / 134 / 264)$ & 70 \\
\hline Total & & & 945 \\
\hline
\end{tabular}

TABLE I: ARHO networks deployed today.

Surprisingly, we see no correlation between environmental conditions and network performance. For example, we see no correlation between the quality of the wireless links and the air temperature or precipitation. Moreover, topographic changes due to snow accumulation or ablation do not affect network performance. We attribute this high performance and resilience, which appears vastly superior to other low-power wireless implementation [7], [17], to the channel hopping nature of a SmartMesh IP network. Due to low-power consumption of the sensor node, the power input from a $10 \mathrm{~W}$ solar panel is sufficient to recharge its battery pack, even under low sunlight conditions during winter. The sensor node battery voltage log, although not presented in this paper, shows no sign of significant current draw during the period the data is collected.

The reliability of the network meets our requirements. The Alpha site, a 37 nodes network, is densely forested in the northern flank and non-forested on the southern flank, with an average canopy coverage of $51 \%$ and an average slope of 15 degrees. 33 out of 37 nodes feature $100 \%$ connectivity between August 2014 and February 2015: they maintain connectivity with the network and continuously report sensor data. The remaining 4 nodes experience a number of hardware and connectivity issue: the charge controller of a sensor node failed, causing it to deplete its battery, another node had a hardware malfunction and dropped from the network, and 2 relay nodes are poorly connected to the network (only a single neighbor), causing it drop from the network from time to time as it cannot exploit path redundancy.

This exemplifies the types of problems we face well, which are always related to either hardware issues, the topography of the terrain, or extreme environmental conditions.

Fig. 4 illustrates the topographical challenges well. Superimposed on Google Earth Photo are the network nodes and links. Because the ARHO networks are deployed in a mountainous region, the terrain often feature sudden elevation drops. Nodes on both sides of the drop have a hard time communicating, and relay nodes need to be placed on the edge as a bridge. Moreover, because of the different elevations, higher gain antenna (e.g. $8 \mathrm{dBi}$ ) often perform less well than lower gain antennas (e.g. $2 \mathrm{dBi}$ ) as the vertical opening of the radiation patter is smaller.

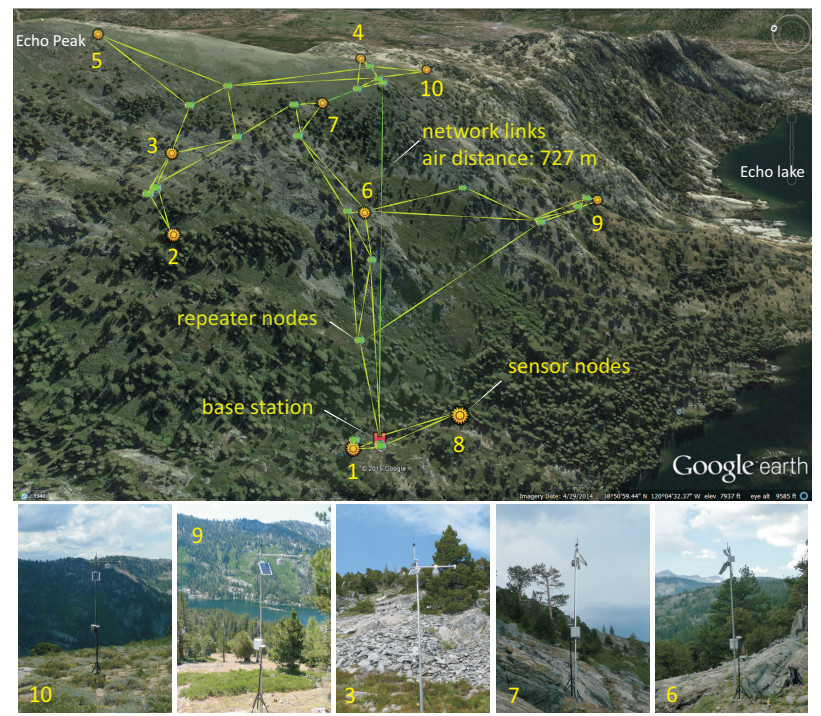

Fig. 4: At site Echo Peak, the sensor nodes location and signal repeater nodes locations as they are in the field are showed on the figure. The lines between the nodes are depicting the actual wireless links made by those elements in the network.

Some ARHO networks, such as Mount Lincoln, are deployed at the top of a mountain, and are therefore exposed to extremely high winds $(150 \mathrm{mph}$ was reported on the Mount Lincoln deployment) In those cases, we reinforced mast and antenna connectors, and used special mounting brackets. On top of wind, water intrusion in the electronics box is problematic. Several sensor nodes on the Van Vleck site had to be replaced in August 2014 after water had damaged the electronics during the 2013-2014 winter season.

\section{Real-Time Dense Hydrometric Data}

We gathered detailed distributed hydrologic and meteorological data from the study sites. Fig. 5 shows a panel of data we gathered from site Alpha. In-situ measurement of snow, air temperature, and relative humidity were gathered and transmitted from our sensor nodes. Five out of ten nodes were also equipped with soil moisture sensor at two depths. A total incoming solar radiation sensor captures the solar energy input. Each node in the network captured sets of data in 

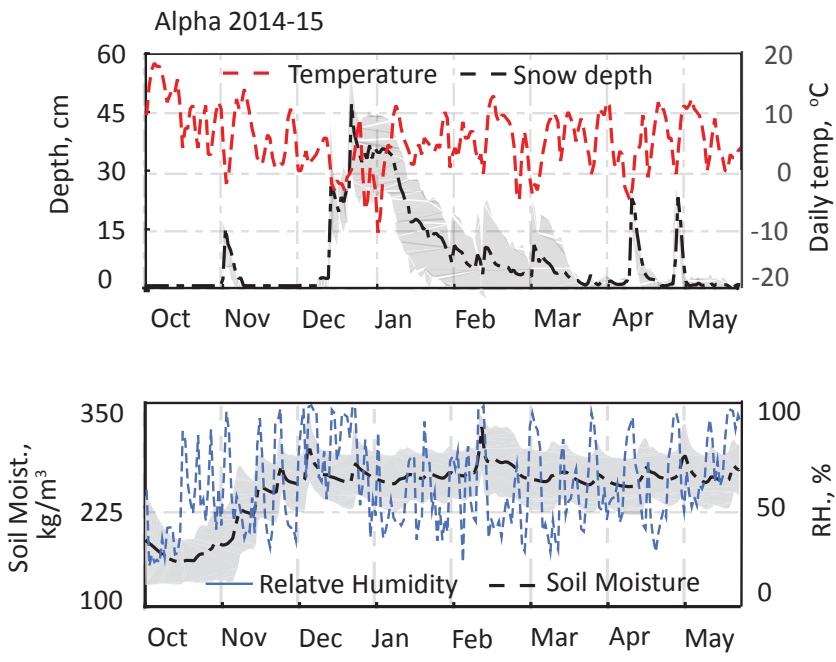

Fig. 5: Snow depth, air temperature, relative humidity and soil moisture data collected at the "Alpha" deployment.

regard to energy and mass fluxes of water in the environment. Measurable and significant variability in all variables are present in our dataset. We are able to quantify those variability at site scale and eventually interpolate across the basin. Data gathered at physiographical representative locations are crucial for developing detailed next generation physical and energy driven models for a better understanding of the processes governs the watershed.

\section{RELATED WORK}

Pushed by the availability of reliable, ultra-low power and cheap devices and related wireless networking technologies, low-power wireless sensors technology is revolutionizing the way environmental sensing is done [18].

Low-power wireless networks are now used to monitor air temperature [19], relative humidity, snow depth [16], [20], soil moisture [21]-[23], permafrost [24], forest fire [25], potential landslides [26] and volcanic activities [27]. Smart Agriculture, in which low-power wireless sensors and actuators monitor and control crops have gained maturity [28]-[30]. Wireless technology for outdoor hydrological monitoring applications are especially enticing as running miles or wires in the woods in unfeasible.

Historically, the study of mountain hydrology and the water cycle has been largely observational, with hydrologic variables extrapolated from a small number of manual measurements [3], [31]-[33]. To partially replace labor-intensive manual collection, meteorological towers have been installed to report a number of environmental readings, typically daily [34]. The technology in those towers is now outdated, and towers can be replaced by large deployments of low-power wireless sensor networks, which produce denser information at a fraction of the cost. Having orders of magnitude more data has helped advance the understanding of watershed science [35].

Several projects use low-power wireless to monitor remote watersheds. In [36] and [17], the authors list the technical challenges in applying this new technology to environmental monitoring. In [37], the authors present a 12-station deployment in a $20 \mathrm{~km}$ catchment of the Swiss Alps - between June and October 2009 - measuring the spacial variability of meteorological forcing parameters such as temperature and precipitation. In [38], 99 sensor-loggers are deployed in three $40-180 \mathrm{~km}^{2}$ basins, to monitor snow cover dynamics in southern Germany. This deployment was carried out for a single winter. Bogena et al. [22] installed 150 wireless nodes connected to over 600 soil moisture sensors in the forest catchment of Wüstebach (Germany) to study spatiotemporal distribution of soil moisture over complex terrain. Over 300 sensors hosted by 60 wireless nodes are deployed at the Southern Sierra Critical Zone Observatory to study complex interaction of water within the snow pack, canopy and soil [16].

ARHO, developed for long term watershed monitoring, is the largest of its kind. It provides real-time insight of the watershed condition. The machine-to-machine capability give bidirectional access to the equipment in the field from the web for debugging, software maintenance and software update.

\section{DISCUSSION}

The size of the American River Basin and the number of devices deployed as part of the ARHO project confirm the clear advantages of using low-power wireless technology over a wired solution. The idea of using an off-the-shelf low-power wireless solution has proven a successful one, although the ARHO project has demonstrated that the networking aspect is only a very small piece of the challenge.

This project has highlighted importance of network monitoring and management tools. Although the SmartMesh IP manager offers a complete interface to query the state of the network, receive alerts, and verify the performance of the network, what is missing is a multi-network management interface. Such a solution would complement an existing SmartMesh IP network by offering a unified interface to assist during network deployment, visualize the network, run network health routines, display the sensor data, and log maintenance activity. We are currently developing this tool through the REALMS associate team ${ }^{9}$, and plan on deploying it within 6 months.

The goal of the first stage of the ARHO project is to gather the sensor data. Describing this first stage has been the focus of this article. The second stage of the project, and our immediate future work, aims at using the sensor data to answer the following mountain hydrology questions. How do physiographic and ecological patterns influence the variability of mountain water fluxes? How do hydrologic systems that are subjected to multiple perturbations respond? How do pulses and changes propagate through the hydrologic system? How can the predictive ability for these responses be improved? In parallel, we are actively looking for opportunities to replicate this work in other parts of the world, and/or applied to other environmental monitoring problems.

\footnotetext{
${ }^{9}$ https://realms-team.github.io/
} 


\section{ACKNOWLEDGMENTS}

The work presented in this paper is supported by the National Science Foundation (NSF) through a Major Research Instrumentation Grant (EAR-1126887), the Southern Sierra Critical Zone Observatory (EAR-0725097), and Inria, through the REALMS associate team.

\section{REFERENCES}

[1] V. Gungor, B. Lu, and G. Hancke, "Opportunities and Challenges of Wireless Sensor Networks in Smart Grid," IEEE Transactions on Industrial Electronics, vol. 57, no. 10, pp. 3557-3564, October 2010.

[2] J. Sen, "A Survey on Wireless Sensor Network Security," International Journal of Communication Networks and Information Security (IJCNIS), vol. 1, no. 2, pp. 55-78, 2009.

[3] R. Rice and R. C. Bales, "Embedded-Sensor Network Design for Snow Cover Measurements around Snow Pillow and Snow Course Sites in the Sierra Nevada of California," Water Resources Research, vol. 46, pp. 1-13, 2010.

[4] Y. Kim, R. G. Evans, and W. M. Iversen, "Remote Sensing and Control of an Irrigation System Using a Distributed Wireless Sensor Network," IEEE Transactions on Instrumentation and Measurement, vol. 57, no. 7, pp. 1379-1387, July 2008.

[5] IEEE802.15.4-2011: Low-Rate Wireless Personal Area Networks (LRWPANs), IEEE Computer Society Std., Rev. IEEE Std 802.15.4-2011, 5 September 2011.

[6] V. Gungor and G. Hancke, "Industrial Wireless Sensor Networks: Challenges, Design Principles, and Technical Approaches," IEEE Transactions on Industrial Electronics, vol. 56, no. 10, pp. 4258-4265, 2009.

[7] P. Huang, L. Xiao, S. Soltani, M. Mutka, and N. Xi, "The Evolution of MAC Protocols in Wireless Sensor Networks: A Survey," IEEE Communications Surveys and Tutorials, vol. 15, no. 1, pp. 101-120, February 2012.

[8] T. Watteyne, L. Doherty, J. Simon, and K. Pister, "Technical Overview of SmartMesh IP," in International Workshop on Extending Seamlessly to the Internet of Things (esIoT), Taiwan, 3-5 July 2013.

[9] T. Watteyne, J. Weiss, L. Doherty, and J. Simon, "Industrial IEEE802.15.4e Networks: Performance and Trade-offs," in International Conference on Communications (ICC). London, UK: IEEE, 8-12 June 2015.

[10] N. Accettura and G. Piro, "Optimal and Secure Protocols in the IETF 6TiSCH Communication Stack," in International Symposium on Industrial Electronics (ISIE). IEEE, 2014, pp. 1469-1474.

[11] A. Berger, M. Pichler, W. Haslmayr, and A. Springer, "Energy Efficient and Reliable Wireless Sensor Networks - An Extension to IEEE 802.15.4e," EURASIP Journal on Wireless Communications and Networking, vol. 126, no. 1, pp. 1-12, 2014.

[12] L. Doherty, J. Simon, and T. Watteyne, "Wireless Sensor Networks: Challenges and Solutions," Microwave Journal, vol. 2012, no. 1, pp. 22-34, August 2012.

[13] M. A. Mahmood, W. K. Seah, and I. Welch, "Reliability in Wireless Sensor Networks: A Survey and Challenges Ahead," Computer Networks, vol. 79, pp. 166-187, March 2015.

[14] K.-H. Phung, B. Lemmens, M. Goossens, A. Nowed, L. Tran, and K. Steenhaut, "Schedule-Based Multi-Channel Communication in Wireless Sensor Networks: A Complete Design and Performance Evaluation," Ad Hoc Networks, vol. 26, pp. 88-102, 2015.

[15] T. Watteyne, Machine-to-machine (M2M) Communications, Architecture, Performance and Applications. Woodhead Publishing, 2015, ch. Lower-power Wireless Mesh Networks for Machine-to-Machine Communications Using the IEEE802.15.4 Standard, pp. 63-79.

[16] B. Kerkez, S. D. Glaser, R. C. Bales, and M. W. Meadows, "Design and Performance of a Wireless Sensor Network for Catchment-Scale Snow and Soil Moisture Measurements," Water Resources Research, vol. 48, no. July 2011, pp. 1-18, 2012.

[17] R. Szewczyk, J. Polastre, A. Mainwaring, and D. Culler, "Lessons from a Sensor Network Expedition," in European Conference on Wireless Sensor Networks (EWSN), vol. 2920. Berlin, Germany: Springer Berlin Heidelberg, 19-21 January 2004, pp. 307-322.

[18] J. Yick, B. Mukherjee, and D. Ghosal, "Wireless Sensor Network Survey," Elsevier Computer Networks, vol. 52, no. 12, pp. 2292-2330, 2008.

[19] J. D. Lundquist and D. R. Cayan, "Surface Temperature Patterns in Complex Terrain: Daily Variations and Long-term Change in the Central Sierra Nevada, California," Journal of Geophysical Research: Atmospheres, vol. 112, no. 11, pp. 1-15, 2007.
[20] A. Varhola, J. Wawerla, M. Weiler, N. C. Coops, D. Bewley, and Y. Alila, "A New Low-cost, Stand-alone Sensor System for Snow Monitoring," Journal of Atmospheric and Oceanic Technology, vol. 27, no. 12, pp. 1973-1978, 2010.

[21] R. C. Bales, J. W. Hopmans, A. T. O'Geen, M. Meadows, P. C. Hartsough, P. Kirchner, C. T. Hunsaker, and D. Beaudette, "Soil Moisture Response to Snowmelt and Rainfall in a Sierra Nevada Mixed-Conifer Forest," Vadose Zone Journal, vol. 10, pp. 786-799, 2011.

[22] H. R. Bogena, M. Herbst, J. Huisman, U. Rosenbaum, A. Weuthen, and H. Vereecken, "Potential of Wireless Sensor Networks for Measuring Soil Water Content Variability," Vadose Zone Journal, vol. 9, no. 4, pp. 1002-1013, 2010.

[23] Z. Li, N. Wang, A. Franzen, C. Taher, P.and Godsey, H. Zhang, and X. Li, "Practical Deployment of an In-Field Soil Property Wireless Sensor Network," Computer Standards \& Interfaces, vol. 36, no. 2, pp. 278-287, February 2014.

[24] A. Hasler, I. Talzi, C. Tschudin, and S. Gruber, "Wireless Sensor Networks in Permafrost Research: Concept, Requirements, Implementation and Challenges," in International Conference on Permafrost (NICOP), 2008, pp. 669-674.

[25] C. Hartung, R. Han, C. Seielstad, and S. Holbrook, "FireWxNet: A Multi-Tiered Portable Wireless System for Monitoring Weather Conditions in Wildland Fire Environments," in International Conference on Mobile Systems, Applications, and Services (MobiSys). Uppsala, Sweden: ACM, 19-22 June 2006

[26] A. Terzis, A. Anandarajah, K. Moore, and I.-J. Wang, "Slip Surface Localization in Wireless Sensor Networks for Landslide Prediction," in International Conference on Information Processing in Sensor Networks (IPSN). Nashville, TN, USA: IEEE, 19-21 April 2006, pp. 109-116.

[27] G. Werner-Allen, K. Lorincz, M. Welsh, O. Marcillo, J. Johnson, M. Ruiz, and J. Lees, "Deploying a Wireless Sensor Network on an Active Volcano," IEEE Internet Computing, vol. 10, no. 2, pp. 18-25, 2006.

[28] R. Beckwith, D. Teibel, and P. Bowen, "Report from the Field: Results from an Agricultural Wireless Sensor Network," in International Conference on Local Computer Networks ( $L C N)$. Tampa, FL, USA: IEEE, 16-18 December 2004, pp. 471-478.

[29] R. Evans and W. Iversen, "Remote Sensing and Control of an Irrigation System Using a Distributed Wireless Sensor Network," IEEE Transactions on Instrumentation and Measurement, vol. 57, no. 7, pp. 13791387,2008

[30] J. Gutierrez, J. F. Villa-Medina, A. Nieto-Garibay, and M. A. PortaGandara, "Automated Irrigation System Using a Wireless Sensor Network and GPRS Module," IEEE Transactions on Instrumentation and Measurement, vol. 63, no. 1, pp. 166-176, 2014.

[31] J. D. Lundquist, D. R. Cayan, and M. D. Dettinger, "Meteorology and Hydrology in Yosemite National Park : A Sensor Network Application," in International Conference on Information Processing in Sensor Networks (IPSN). Palo Alto, CA, USA: ACM, 22-23 April 2003.

[32] M. S. Raleigh and J. D. Lundquist, "Comparing and Combining SWE Estimates from the SNOW-17 Model using PRISM and SWE Reconstruction," Water Resources Research, vol. 48, no. 1, pp. 1-16, January 2012.

[33] C. Res, C. Daly, W. P. Gibson, G. H. Taylor, G. L. Johnson, and P. Pasteris, "A Knowledge-based Approach to the Statistical Mapping of Climate," Climate Research, vol. 22, no. 2, pp. 99-113, 2002.

[34] C. Daly, M. Halbleib, J. I. Smith, W. P. Gibson, M. K. Doggett, G. H. Taylor, and P. P. Pasteris, "Physiographically Sensitive Mapping of Climatological Temperature and Precipitation across the Conterminous United States," International Journal of Climatology, vol. 28, no. 15, pp. 2031-2064, December 2008

[35] R. C. Bales, N. P. Molotch, T. H. Painter, M. D. Dettinger, R. Rice, and J. Dozier, "Mountain Hydrology of the Western United States," Water Resources Research, vol. 42, no. 8, pp. 1-13, 2006

[36] A. Mainwaring, D. Culler, J. Polastre, R. Szewczyk, and J. Anderson, "Wireless Sensor Networks for Habitat Monitoring," in International Workshop on Wireless Sensor Networks and Applications (WSNA). Atlanta, GA, USA: ACM, 28 September 2002, pp. 88-97.

[37] S. Simoni, S. Padoan, D. Nadeau, M. Diebold, A. Porporato, G. Barrenetxea, F. Ingelrest, M. Vetterli, and M. Parlange, "Hydrologic Response of an Alpine Watershed : Application of a Meteorological Wireless Sensor Network to Understand Streamflow Generation," Water Resources Research, vol. 47, no. 10, pp. 1-16, 2011.

[38] S. Pohl, J. Garvelmann, , J. Wawerla, and M. Weiler, "Potential of a Lowcost Sensor Network to Understand the Spatial and Temporal Dynamics of a Mountain Snow Cover," Water Resources Research, vol. 50, no. 3, pp. 2533-2550, March 2014. 


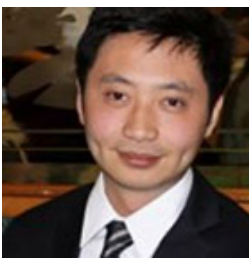

Ziran Zhang is an outdoor enthusiast and a $\mathrm{PhD}$ student at the University of California, Berkeley, in the Department of Civil and Environmental Engineering, working with Prof. Steven Glaser. He is the student lead of team which designs, builds and deploys low-power wireless sensors for environmental monitoring. In the American River Hydrology Observatory (ARHO) project, funded by the NSF, he uses 945 low-power wireless sensors deployed across the American River Basin to monitor the snowpack in California's Sierra Nevada Mountains, in real-time. His research interests include applications of wireless sensor networks, data analytics and data mining and cyber-physical system design. In his leisure time, Ziran enjoys exploring the nature and foraging for rare kitchen ingredients. Ziran holds a BSc (2012) and MSc (2013) degrees from the University of California, Berkeley.

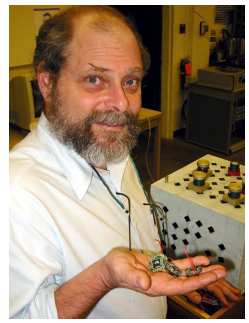

Steven Glaser is a Professor of Systems Engineering in the Department of Civil and Environmental Engineering at the University of California, Berkeley. He is a founding member of the Center for Information Technology Research in the Interest of Society. His specialties include wireless sensor networks, measuring things, laboratory earthquakes, and snow hydrology for hydroelectric generation. In addition to his engineering education he holds a BA in Philosophy.

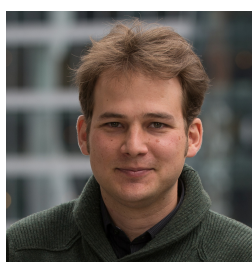

Thomas Watteyne is an insatiable enthusiast of lowpower wireless mesh technologies. He is a researcher at Inria in Paris, in the new EVA research team, where he designs, models and builds networking solutions based on a variety of Internet-of-Things (IoT) standards. He is Senior Networking Design Engineer at Linear Technology, in the Dust Networks product group, the undisputed leader in supplying low power wireless mesh networks for demanding industrial process automation applications. Since 2013, he cochairs the IETF 6TiSCH working group, which standardizes how to use IEEE802.15.4e TSCH in IPv6-enabled mesh networks, and recently joined the IETF Internet-of-Things Directorate. Prior to that, Thomas was a postdoctoral research lead in Prof. Kristofer Pister's team at the University of California, Berkeley. He founded and co-leads Berkeley's OpenWSN project, an open-source initiative to promote the use of fully standards-based protocol stacks for the IoT. Between 2005 and 2008, he was a research engineer at France Telecom, Orange Labs. He holds a $\mathrm{PhD}$ in Computer Science (2008), an MSc in Networking (2005) and an MEng in Telecommunications (2005) from INSA Lyon, France. He is Senior member of IEEE. He is fluent in 4 languages.

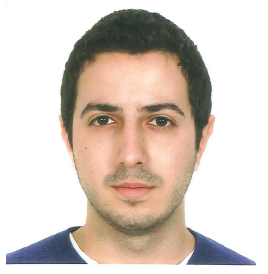

Sami Malek obtained his B.E. degree in Electrical and Computer Engineering with high distinction from the American University of Beirut, Lebanon. He recently joined the Civil Systems department at $\mathrm{UC}$ Berkeley as $\mathrm{MS} / \mathrm{PhD}$ student where he completed his Masters. He is currently contributing in the development and installation of large-scale Wireless Sensor Networks in the Sierra Mountains. The NSF-funded project aims at collecting realtime hydrologic data for improved forecasting and optimal resource management. His current interests include embedded systems, networking, communications and machine learning. Recipient of the IEEE Student Enterprise Award and the AUB Creative Achievement Award, Sami finds his utmost productivity in challenging and mind-thrilling projects. He plays guitar and is said to be talented in drawing. 